\title{
A Comparison Theorem for Nonsmooth Nonlinear Operators
}

\author{
Vladimir Kozlov ${ }^{1}$ (D) . Alexander Nazarov ${ }^{2,3}$
}

Received: 25 January 2019 / Accepted: 2 February 2020 / Published online: 11 March 2020

(C) The Author(s) 2020

\begin{abstract}
We prove a comparison theorem for super- and sub-solutions with non-vanishing gradients to semilinear PDEs provided a nonlinearity $f$ is $L^{p}$ function with $p>1$. The proof is based on a strong maximum principle for solutions of divergence type elliptic equations with VMO leading coefficients and with lower order coefficients from a Kato class. An application to estimation of periodic water waves profiles is given.
\end{abstract}

Keywords Semi-linear elliptic equation · Non-smooth nonlinearity ·

Comparison principal · VMO coefficients $\cdot$ Kato classes $\cdot$ Strong maximum principle

Mathematics Subject Classification (2010) 35J61 · 35B50 · 35B51 · 35J15

\section{Introduction}

Let $\Omega$ be a domain in $\mathbb{R}^{n}, n \geq 2$. We will consider super- and sub-solutions of the equation

$$
\Delta u+f(u)=0 \text { in } \Omega,
$$

where $f$ is a real valued function from $L_{\text {loc }}^{p}(\mathbb{R})$ with some $p>1$. To make the term $f(u)$ well-defined (measurable and belonging to $L_{\mathrm{loc}}^{p}(\Omega)$ ) we will assume that $u \in C^{1}(\Omega)$ and $\nabla u \neq 0$ in $\Omega$. Usually, $\mathrm{f}$ is supposed to be continuous in almost all papers dealing with Eq. 1 and its generalisations (see, for example, [6] and [11] and numerous papers citing these notes).

It was shown in [4, Remark 2.3] that the strong maximum principle may fail if the function $f$ is only Hölder continuous with an exponent less than 1. Optimal conditions on smoothness of $f$ for validity of the strong maximum principle can be found in [12]. The main difference in our approach is that we compare functions in a neughborhood of a point

Vladimir Kozlov

vladimir.kozlov@liu.se

1 Department of Mathematics, University of Linköping, Linköping SE-581 83, Sweden

2 St.-Petersburg Department of Steklov Mathematical Institute, Fontanka, 27, St.-Petersburg, 191023, Russia

3 St.-Petersburg State University, Universitetskii pr. 28, St.-Petersburg, 198504, Russia 
where the gradients are not vanishing. This allows to remove any smoothness assumptions on $f$.

One of the main results of this paper is the following assertion:

Theorem 1.1 Let $f \in L_{\text {loc }}^{p}(\Omega), p>1$. Also let $u_{1}, u_{2} \in C^{1}(\Omega)$ have non-vanishing gradients in $\Omega$ and satisfy the inequalities

$$
\Delta u_{1}+f\left(u_{1}\right) \geq 0 \text { and } \Delta u_{2}+f\left(u_{2}\right) \leq 0
$$

in the weak sense. If $u_{1} \leq u_{2}$ and $u_{1}\left(x_{0}\right)=u_{2}\left(x_{0}\right)$ for some $x_{0} \in \Omega$ then $u_{1}=u_{2}$ in the whole $\Omega$.

We note that the theorem is not true without assumptions that the gradients do not vanish, which follows from [4] (see [7]).

In the case $p>n$ this theorem was proved in [7]. The proof there was based on a weak Harnack inequality for non-negative solutions to the second order equation in divergence form

$$
\mathcal{L} u:=D_{j}\left(a_{j i}(x) D_{i} u\right)+b_{j}(x) D_{j} u=0 \quad \text { in } \quad \Omega
$$

and closely connected with $L^{p}$ properties of the coefficients $b_{j}$. Therefore one of our main concerns is a strong maximum principle for solutions to Eq. 3. We always assume that the matrix $\left(a_{i j}\right)$ is symmetric and uniformly elliptic:

$$
v|\xi|^{2} \leq a_{i j}(x) \xi_{i} \xi_{j} \leq v^{-1}|\xi|^{2}, \quad x \in \Omega, \xi \in \mathbb{R}^{n} .
$$

It was proved in [14] that if $|b| \in L_{\mathrm{loc}}^{p}(\Omega)$ (here $b=\left(b_{1}, \ldots, b_{n}\right)$ ) with $p>n$ then a nonnegative weak solution to Eq. 3 satisfies (here $B_{\rho}(x)$ stands for the ball of radius $\rho$ centered at $x$ )

$$
\rho^{-n / \gamma}\|u\|_{L^{\gamma}\left(B_{2 \rho}\left(x^{0}\right)\right)} \leq C \inf _{x \in B_{\rho}\left(x^{0}\right)} u(x),
$$

where $B_{3 \rho}\left(x^{0}\right) \subset \Omega$ and $\gamma \in(1, n /(n-2))$. So the restriction $p>n$ in this assertion inherits in the theorem in [7]. ${ }^{1}$

For our purpose another type of assumptions on the coefficients $b_{j}$ are more appropriate. It is called the Kato condition, see [3] and [13].

Definition 1 We say that $f \in K_{n, \alpha}$ if

$$
\sup _{x} \int_{|x-y| \leq r} \frac{|f(y)|}{|x-y|^{n-\alpha}} d y \rightarrow 0 \quad \text { as } \quad r \rightarrow 0 .
$$

It was proved in [8] that inequality (4) still holds if $|b|^{2} \in K_{n, 2}$. For Hölder continuous coefficients $a_{j i}$ (4) was proved in [15] under the assumption $|b| \in K_{n, 1}$. We note that from the last assertion it follows (4) when $|b| \in L_{\text {loc }}^{p}, p>1$, depends only on one variable and the leading coefficients are Hölder continuous.

For our applications we need the leading coefficients to be only continuous. So all above mentioned results are not enough for our purpose. Here we prove a theorem which deals with slightly discontinuous leading coefficients and allows $b^{\alpha} \in K_{n, \alpha}$ with $\alpha$ close to 1 for lower order coefficients. In order to formulate this result we need some definitions.

\footnotetext{
${ }^{1}$ It was pointed out in [10, Theorem 2.5'] that Eq. 4 holds if $|b| \log ^{1 / 2}(1+|b|) \in L_{\text {loc }}^{2}(\Omega)$ for $n=2$ and if $|b| \in L_{\text {loc }}^{n}(\Omega)$ for $n \geq 3$.
} 
Definition 2 Let $f(x)$ be a measurable and locally integrable function. Define a quantity

$$
f^{\# r}(x):=\frac{1}{\left|B_{r}\right|} \int_{B_{r}(x)}\left|f(y)-\frac{1}{\left|B_{r}\right|} \int_{B_{r}(x)} f(z) d z\right| d y ; \quad \omega_{f}(\rho)=\sup _{x} \sup _{r \leq \rho} f^{\# r}(x) .
$$

We say that $f \in V M O\left(\mathbb{R}^{n}\right)$ if $\omega_{f}(\rho)$ is bounded and $\omega_{f}(\rho) \rightarrow 0$ as $\rho \rightarrow 0$. In this case the function $\omega_{f}(\rho)$ is called VMO-modulus of $f$.

For a bounded Lipschitz domain $\Omega$ the space $f \in V M O(\Omega)$ is introduced in the same way but the integrals in the definition of $f^{\# r}(x)$ are taken over $B_{r}(x) \cap \Omega$.

Definition 3 We say that a function $\sigma:[0,1] \rightarrow \mathbb{R}_{+}$belongs to the Dini class $\mathcal{D}$ if $\sigma$ is increasing, $\sigma(t) / t$ is summable and decreasing.

It should be noted that assumption about the decay of $\sigma(t) / t$ is not restrictive (see Remark 1.2 in [1] for more details). We use the notation $\varkappa(\rho)=\int_{0}^{\rho} \frac{\sigma(t)}{t} d t$.

Theorem 1.2 Let $n \geq 3$. Assume that the leading coefficients $a_{i j} \in V M O(\Omega)$. Suppose that $|b|^{\alpha} \in K_{n, \alpha}$ and

$$
\sup _{x} \int_{r / 2 \leq|x-y| \leq r} \frac{|b(y)|^{\alpha}}{|x-y|^{n-\alpha}} d y \leq \sigma^{\alpha}(r)
$$

for some $\alpha>1$ and $\sigma \in \mathcal{D}$.

If a function $u \in W^{1, p}(\Omega), p>n$, satisfies $u \geq 0$ and $\mathcal{L} u \geq 0$ in $\Omega$ then either $u>0$ in $\Omega$ or $u \equiv 0$ in $\Omega$.

Remark 1 Notice that the assumption $|b|^{\alpha_{2}} \in K_{n, \alpha_{2}}$ does not imply $|b|^{\alpha_{1}} \in K_{n, \alpha_{1}}$ for any $1<\alpha_{1}<\alpha_{2}$. However, if the condition (6) holds with $\alpha=\alpha_{2}$ then the Hölder inequality ensures $|b|^{\alpha_{1}} \in K_{n, \alpha_{1}}$, and Eq. 6 holds with $\alpha=\alpha_{1}$ (and another function $\sigma \in \mathcal{D}$ ).

For $n=2$ we need a stronger assumption.

Theorem 1.3 Let $n=2$. Assume that the leading coefficients $a_{i j} \in V M O(\Omega)$. Suppose that

$$
\sup _{x} \int_{r / 2 \leq|x-y| \leq r} \frac{|b(y)|^{\alpha} \log ^{\alpha} \frac{r}{|x-y|}}{|x-y|^{2-\alpha}} d y \leq \sigma^{\alpha}(r)
$$

for some $\alpha>1$ and $\sigma \in \mathcal{D}$.

If a function $u \in W^{1, p}(\Omega), p>2$, satisfies $u \geq 0$ and $\mathcal{L} u \geq 0$ in $\Omega$ then either $u>0$ in $\Omega$ or $u \equiv 0$ in $\Omega$.

For $\gamma \in(0,1)$ we define the annulus

$$
\mathcal{X}_{r, \gamma}(x)=\left\{y \in B_{r}(x):|x-y|>\gamma r\right\}
$$

If the location of the center is not important we write simply $B_{r}$ and $\mathcal{X}_{r, \gamma}$. 
As usual, for a bounded domain $\Omega$ we denote by $W_{0}^{1, q}(\Omega), q>1$, the closure in $W^{1, q}(\Omega)$ of the set of smooth compactly supported function, with the norm

$$
\|u\|_{W_{0}^{1, q}(\Omega)}=\left(\int_{\Omega}|\nabla u|^{q} d x\right)^{1 / q} .
$$

\section{Strong Maximum Principle for Operators with Lower Order Terms}

\subsection{Coercivity}

Let $\Omega^{\prime}$ be a bounded subdomain in $\Omega$. Consider the problem

$$
\mathcal{L}_{0} u:=D_{j}\left(a_{i j}(x) D_{i} u\right)=f \quad \text { in } \quad \Omega^{\prime} ; \quad u=0 \quad \text { on } \quad \partial \Omega^{\prime} .
$$

We say that the operator $\mathcal{L}_{0}$ is $q$-coercive in $\Omega^{\prime}$ for some $q>1$, if for each $f \in W^{-1, q}\left(\Omega^{\prime}\right)$ the problem (9) has a unique solution $u \in W_{0}^{1, q}\left(\Omega^{\prime}\right)$ and this solution satisfies

$$
\|u\|_{W_{0}^{1, q}\left(\Omega^{\prime}\right)} \leq C_{q}\|f\|_{W^{-1, q}\left(\Omega^{\prime}\right)}
$$

with $C_{q}$ independent on $f$ and $u$.

It is well known that for arbitrary measurable and uniformly elliptic coefficients the operator $\mathcal{L}_{0}$ is 2-coercive in arbitrary bounded $\Omega^{\prime}$. Further, if the coefficients $a_{i j} \in \operatorname{VMO}(\Omega)$ then the operator $\mathcal{L}_{0}$ is $q$-coercive for arbitrary $q>1$ in arbitrary bounded $\Omega^{\prime} \subset \Omega$ with $\partial \Omega^{\prime} \in \mathcal{C}^{1}$, see [2]. The coercivity constant $C_{q}$ depends on $\Omega^{\prime}$ and VMO-moduli of $a_{i j}$. Moreover, by dilation we can see that for $\Omega^{\prime}=B_{r}, r \leq 1$, this constant does not depend on $r$. For $\Omega^{\prime}=\mathcal{X}_{r, \gamma}, r \leq 1, C_{q}$ depends on $\gamma$ but not on $r$.

Let now the operator in Eq. 9 be $q$-coercive for certain $q>2$. Put

$$
f=\nabla \cdot \mathbf{f}+f_{0},
$$

where $\mathbf{f}=\left(f_{1}, \ldots, f_{n}\right) \in\left(L^{q}\left(\Omega^{\prime}\right)\right)^{n}$ and $f_{0} \in L^{n q /(n+q)}\left(\Omega^{\prime}\right)$. Then by the imbedding theorem $f \in W^{-1, q}\left(\Omega^{\prime}\right)$ and Eq. 10 takes the form

$$
\|u\|_{W_{0}^{1, q}\left(\Omega^{\prime}\right)} \leq C\left(\sum_{j=1}^{n}\left\|f_{j}\right\|_{L^{q}\left(\Omega^{\prime}\right)}+\left\|f_{0}\right\|_{L^{n q /(n+q)}\left(\Omega^{\prime}\right)}\right) .
$$

We need the following local estimate.

Theorem 2.1 Let $\Omega^{\prime}$ be a bounded Lipschitz subdomain of $\Omega$ and let the operator in Eq. 9 be $q$-coercive for certain $q>2$. Let also $u \in W^{1, q}\left(\Omega^{\prime}\right)$ be such that

$$
D_{j}\left(a_{j i}(x) D_{i} u\right)=0 \quad \text { in } \quad \Omega^{\prime} \cap B_{r} ; \quad u=0 \quad \text { on } \quad \partial \Omega^{\prime} \cap B_{r} .
$$

Then for a fixed $\lambda \in(0,1)$

$$
\|\nabla u\|_{L^{q}\left(B_{\lambda r} \cap \Omega^{\prime}\right)} \leq C r^{-1}\|u\|_{L^{q}\left(B_{r} \cap \Omega^{\prime}\right)},
$$

where $C$ may depend on the domain $\Omega^{\prime}, q, \lambda$ and the coercivity constant $C_{q}$ but it is independent of $r$.

Proof First, we claim that the problem (9) is $s$-coercive for any $s \in[2, q]$. Indeed, we have coercivity for $s=2$ and $s=q$, and the claim follows by interpolation. 
Second, the estimate (11) for $q=2$ follows by the the Caccioppoli inequality. For $q>2$ we choose a cut-off function $\zeta$ such that $\zeta=1$ on $B_{\lambda r}$ and $\zeta=0$ outside $B_{\lambda_{1} r}$, where $\lambda<\lambda_{1}<1$, and $\nabla \zeta \leq c r^{-1}$. Then

$$
D_{j}\left(a_{j i}(x) D_{i}(\zeta u)\right)=D_{j}\left(a_{j i}(x) u D_{i} \zeta\right)+\left(D_{j} \zeta\right) a_{j i}(x) D_{i} u \quad \text { in } \quad \Omega^{\prime} ; \quad \zeta u=0 \quad \text { on } \quad \partial \Omega^{\prime} .
$$

Then by the $s$-coercivity of the operator we have

$$
\|\nabla u\|_{L^{s}\left(B_{\lambda r}\right)} \leq C r^{-1}\left(\|u\|_{L^{s}\left(B_{\lambda_{1}} r\right)}+\|\nabla u\|_{L^{n s /(n+s)}\left(B_{\lambda_{1} r}\right)}\right)
$$

We choose $s=\min (q, 2 n /(n-2))$. Then the last term in the right-hand side is estimated by $C r^{n / s-n / 2}\|\nabla u\|_{L^{2}\left(B_{\lambda_{1} r}\right)}$, and hence by the proved estimate for $q=2$, we obtain (11) for $q=s$. Repeating this argument (but using now the estimate (11) for $q=s$ ) we arrive finally at Eq. 11.

\subsection{Estimates of the Green Functions}

Let $\mathcal{L}$ be an operator of the form Eq. 3, and the assumptions of Theorem 1.2 are fulfilled. We establish the existence and some estimates of the Green function $G=G(x, y)$ for the problem

$$
\mathcal{L} u=f \quad \text { in } \quad B_{r} ; \quad u=0 \quad \text { on } \quad \partial B_{r},
$$

in sufficiently small ball $B_{r} \subset \Omega$.

Lemma 2.1 Let $n \geq 3$. There exists a positive constant $R$ depending on $n$, the ellipticity constant $\nu, V M O$-moduli of coefficients $a_{i j}$, the exponent $\alpha$ and the function $\sigma$ from Eq. 6 such that for any $r \leq R$ there is the Green function $G(x, y)$ of the problem (12) in a ball $B_{r} \subset \Omega$. Moreover, it is continuous w.r.t. $x$ for $x \neq y$ and satisfies the estimates

$$
\begin{aligned}
& \quad|G(x, y)| \leq \frac{C_{1}}{|x-y|^{n-2}} \\
& \text { if }|x-y| \leq \operatorname{dist}\left(x, \partial B_{r}\right) / 2 \quad \text { then } \quad G(x, y) \geq \frac{C_{2}}{|x-y|^{n-2}},
\end{aligned}
$$

where the constants $C_{1}$ and $C_{2}$ depend on the same quantities as $R$.

Proof We use the idea from [15]. Denote by $G_{0}(x, y)$ the Green function of the problem (9) in the ball $B_{r}$. The estimates (13) for $G_{0}$ were proved in [9] (see also [5]):

$$
\begin{aligned}
& 0<G_{0}(x, y) \leq \frac{C_{10}}{|x-y|^{n-2}} \\
& \text { if }|x-y| \leq \operatorname{dist}\left(x, \partial B_{r}\right) / 2 \quad \text { then } \quad G_{0}(x, y) \geq \frac{C_{20}}{|x-y|^{n-2}},
\end{aligned}
$$

where the constants $C_{10}$ and $C_{20}$ depend only on $n$ and $v$. 
By Remark 1, we can assume without loss of generality $\alpha<n /(n-1)$. Put $q=\alpha^{\prime}>n$ and denote by $C_{q}$ the coercivity constant for $\mathcal{L}_{0}$ in the ball. We begin with the estimate for any $B_{\rho}(y) \subset \Omega$

$$
\begin{aligned}
& \int_{B_{\rho}(y)}|b(x)|\left|D_{x} G_{0}(x, y)\right| d x=\sum_{j=0}^{\infty} \int_{\mathcal{X}_{\frac{\rho}{2^{j}, \frac{1}{2}}}(y)}|b(x)|\left|D_{x} G_{0}(x, y)\right| d x \\
\leq & \left.\left.\sum_{j=0}^{\infty}\left(\int_{\mathcal{X}_{\frac{\rho}{2^{j}}, \frac{1}{2}}(y)} \mid D_{x} G_{0}(x, y)\right)\right|^{q} d x\right)^{\frac{1}{q}}\left(\int_{\mathcal{X}_{\frac{\rho}{2^{j}}, \frac{1}{2}}(y)}|b(x)|^{q^{\prime}} d x\right)^{\frac{1}{q^{\prime}}}=: \sum_{j=0}^{\infty} A_{j 1} A_{j 2 .}
\end{aligned}
$$

By Eqs. 11 and 14, we have $A_{j 1} \leq C\left(n, v, C_{q}\right)\left(2^{-j-1} \rho\right)^{1-\frac{n}{q^{\prime}}}$, and Eq. 6 gives

$$
A_{j 1} A_{j 2} \leq 2^{\frac{n}{q^{\prime}}-1} C\left(\int_{\mathcal{X}_{\frac{\rho}{2 j}, \frac{1}{2}}(y)} \frac{|b(x)|^{q^{\prime}}}{|x-y|^{n-q^{\prime}}} d x\right)^{\frac{1}{q^{\prime}}} \leq 2^{\frac{n}{q^{\prime}}-1} C \sigma\left(2^{-j} \rho\right) .
$$

Therefore,

$$
\int_{B_{\rho}(y)}|b(x)|\left|D_{x} G_{0}(x, y)\right| d x \leq 2^{\frac{n}{q^{\prime}}-1} C \sum_{j=0}^{\infty} \sigma\left(2^{-j} \rho\right) \leq A\left(n, v, q, C_{q}\right) \int_{0}^{\rho} \frac{\sigma(t)}{t} d t \equiv A \varkappa(\rho) .
$$

Next, we write down the equation for $G$

$$
\mathcal{L}_{0}\left(G-G_{0}\right)=-b \cdot D G \quad \Longleftrightarrow \quad\left(I+G_{0} *(b \cdot D)\right)\left(G-G_{0}\right)=-b \cdot D G_{0}
$$

and obtain

$$
G=G_{0}-G_{0} *\left(b \cdot D G_{0}\right)+G_{0} *\left(b \cdot D G_{0}\right) *\left(b \cdot D G_{0}\right)-\cdots=: \sum_{k=0}^{\infty} J_{k}
$$

provided this series converges.

We claim that

$$
\left|J_{k}(x, y)\right| \leq \frac{C_{10} C^{k} \varkappa^{k}(r)}{|x-y|^{n-2}} \Longrightarrow\left|J_{k+1}(x, y)\right| \leq \frac{C_{10} C^{k+1} \varkappa^{k+1}(r)}{|x-y|^{n-2}},
$$

for a proper constant $C$. Indeed,

$$
J_{k+1}(x, y)=\int_{B_{r}} J_{k}(x, z)\left(b(z) \cdot D_{z} G_{0}(z, y)\right) d z .
$$

Denote $2 \rho=|x-y|$. Then

$$
\left|J_{k+1}(x, y)\right| \leq\left(\int_{B_{r} \backslash B_{\rho}(x)}+\int_{B_{r} \cap B_{\rho}(x)}\right)\left|J_{k}(x, z)\right||b(z)|\left|D_{z} G_{0}(z, y)\right| d z=: I_{1}+I_{2} .
$$


We have by Eq. 15

$$
\begin{aligned}
I_{1} & \leq \int_{B_{r} \backslash B_{\rho}(x)} \frac{C_{10} C^{k} \varkappa^{k}(r)}{|x-z|^{n-2}}\left|\left(b(z)|| D_{z} G_{0}(z, y)\right)\right| d z \\
& \leq \frac{2^{n-2} C_{10} C^{k} \varkappa^{k}(r)}{|x-y|^{n-2}} \int_{B_{r} \backslash B_{\rho}(x)}|b(z)|\left|D_{z} G_{0}(z, y)\right| d z \leq \frac{2^{n-1} A C_{10} C^{k} \varkappa^{k+1}(r)}{|x-y|^{n-2}}
\end{aligned}
$$

(here we used an evident inequality $\varkappa(2 r) \leq 2 \varkappa(r)$ ). Further,

$$
I_{2} \leq \int_{B_{r} \cap B_{\rho}(x)} \frac{A C^{k} \varkappa^{k}(r)}{|x-z|^{n-2}}|b(z)|\left|D_{z} G_{0}(z, y)\right| d z .
$$

By Eqs. 11 and 14,

$$
\left.\left.\left(\int_{B_{r} \cap B_{\rho}(x)} \mid D G_{0}(z, y)\right)\right|^{q} d y\right)^{\frac{1}{q}} \leq A \rho^{1-\frac{n}{q^{\prime}}} .
$$

Using the assumption $q>n$ we get

$$
I_{1} \leq \frac{A^{2} C^{k} \varkappa^{k}(r)}{\rho^{\frac{n}{q^{\prime}}-1}}\left(\int_{B_{r} \cap B_{\rho}(x)} \frac{|b(z)|^{q^{\prime}}}{|x-z|^{(n-2) q^{\prime}}} d z\right)^{\frac{1}{q^{\prime}}} \leq \frac{A^{2} C^{k} \varkappa^{k}(r)}{\rho^{\frac{n}{q^{\prime}}-1}} \rho^{1-\frac{n}{q}} \varkappa(\rho) \leq \frac{2^{n-2} A^{2} C^{k} \varkappa^{k+1}(r)}{|x-y|^{n-2}},
$$

and the claim follows if we put $C=2^{n-2}\left(A+2 C_{10}\right)$.

Thus, the series in Eq. 16 converges if $\varkappa(r)$ is sufficiently small. Moreover, if $2^{n-2}(A+$ $\left.2 C_{10}\right) \varkappa(r) \leq \frac{C_{20}}{C_{20}+2 C_{10}}$ then Eqs. 14 implies 13 with $C_{1}=C_{10}+\frac{C_{20}}{2}, C_{2}=\frac{C_{20}}{2}$.

To prove the continuity of $G$ we take $\widehat{x}$ such that $|x-\widehat{x}| \leq \rho / 2=|x-y| / 4$. Since $q>n$, the estimate (11) and the Morrey embedding theorem give

$$
\left|G_{0}(x, y)-G_{0}(\widehat{x}, y)\right| \leq C_{30}\left(n, v, C_{q}\right) \frac{|x-\widehat{x}|^{1-\frac{n}{q}}}{|x-y|^{\frac{n}{q^{\prime}}-1}} .
$$

We write down the relation

$$
\left.G(x, y)-G_{(} \widehat{x}, y\right)=\sum_{k=0}^{\infty}\left(J_{k}(x, y)-J_{k}(\widehat{x}, y)\right)
$$

and deduce, similarly to Eq. 17, that

$$
\begin{aligned}
& \left|J_{k}(x, y)-J_{k}(\widehat{x}, y)\right| \leq C_{30} C^{k} \varkappa^{k}(r) \frac{|x-\widehat{x}|^{1-\frac{n}{q}}}{|x-y|^{\frac{n}{q^{\prime}}-1}} \\
& \Longrightarrow\left|J_{k+1}(x, y)-J_{k+1}(\widehat{x}, y)\right| \leq C_{30} C^{k+1} \varkappa^{k+1}(r) \frac{|x-\widehat{x}|^{1-\frac{n}{q}}}{|x-y|^{\frac{n}{q^{\prime}}-1}} .
\end{aligned}
$$

Therefore, if $\varkappa(r)$ is sufficiently small,

$$
|G(x, y)-G(\widehat{x}, y)| \leq C_{3} \frac{|x-\widehat{x}|^{1-\frac{n}{q}}}{|x-y|^{\frac{n}{q^{+}}-1}} .
$$

Remark 2 In fact, since $q$ can be chosen arbitrarily large, $G$ is locally Hölder continuous w.r.t. $x$ for $x \neq y$ with arbitrary exponent $\beta \in(0,1)$. 
Now let $\mathcal{X}_{r, \gamma} \subset \Omega$. Consider the Dirichlet problem

$$
\mathcal{L} u=f \quad \text { in } \quad \mathcal{X}_{r, \gamma} ; \quad u=0 \quad \text { on } \quad \partial \mathcal{X}_{r, \gamma} .
$$

Lemma 2.2 The statement of Lemma 2.1 holds for the problem (18). The constants $R, C_{1}$ and $\mathrm{C}_{2}$ may depend on the same quantities as in Lemma 2.1 and also on $\gamma$.

The proof of Lemma 2.1 runs without changes.

\subsection{Approximation Lemma and Weak Maximum Principle}

Lemma 2.3 Under assumptions of Lemma 2.1, let $u$ be a weak solution of the equation

$$
\mathcal{L} u=f \quad \text { in } \quad B_{r} \subset \Omega,
$$

with $r \leq R$ where $R$ is the constant from Lemma 2.1. Let $f$ be a finite signed measure in $B_{r}$.

Put $b_{i}^{m}:=b_{i} \chi_{\{|b| \leq m\}}$ and define a sequence $f_{m} \in L^{\infty}\left(B_{r}\right)$ such that $f_{m} \rightarrow f$ in the space of measures. Denote by $u_{m}$ the solution of the problem

$$
\mathcal{L}_{m} u_{m}:=-D_{i}\left(a_{i j} D_{j} u_{m}\right)+b_{i}^{m} D_{i} u_{m}=f^{m} \quad \text { in } \quad B_{r} ; \quad u^{m}=u \quad \text { on } \quad \partial B_{r} .
$$

Then

$$
\int_{B_{r}}\left|u_{m}(x)-u(x)\right| d x \rightarrow 0 \quad \text { as } \quad m \rightarrow \infty .
$$

Proof It is easy to see that the difference $v_{m}=u_{m}-u$ solves the problem

$$
\mathcal{L}_{m} v_{m}=\left(b_{i}-b_{i}^{m}\right) D_{i} u+f_{m}-f \quad \text { in } \quad B_{r} ; \quad v_{m}=0 \quad \text { on } \partial B_{r} .
$$

Using the Green function $G_{m}$ of the operator $\mathcal{L}_{m}$ in $B_{r}$ with the Dirichlet boundary conditions we get

$$
\int_{B_{r}}\left|v_{m}(x)\right| d x \leq\left(\int_{B_{r}}\left|\left(b_{i}-b_{i}^{m}\right) D_{i} u\right|+\int_{B_{r}}\left|f_{m}-f\right|\right) \cdot \sup _{y} \int_{B_{r}}\left|G_{m}(x, y)\right| d x .
$$

By Lemma 2.1, $\left|G_{m}(x, y)\right| \leq C|x-y|^{2-n}$ with constant independent of $m$. Thus, the supremum of the last integral is bounded. The first integral in brackets tends to zero by the Lebesgue Dominated convergence Theorem, and the Lemma follows.

Corollary 2.1 If $\mathcal{L} u=f \geq 0$ in $B_{r}$, and $u \geq 0$ on $\partial B_{r}$, then $u \geq 0$ in $B_{r}$.

This statement follows from standard weak maximum principle and Lemma 2.3.

Lemma 2.4 Let $\mathcal{X}_{r, \gamma} \subset \Omega$ and let $r \leq R$ where $R$ is the constant from Lemma 2.2. Then the assertion of Lemma 2.3 and the Corollary 2.1 are still true.

\subsection{Strong Maximum Principle}

Lemma 2.5 Let $B_{r} \subset \Omega$ be a ball and let $r \leq R$ where $R$ satisfies the assumptions of Lemma 2.1 and Lemma 2.2 with $\gamma=1 / 4$. Then the Green function of $\mathcal{L}$ in $B_{r}$ is strictly positive: $G(x, y)>0$ for $x, y \in B_{r}, x \neq y$. 
Proof First suppose that $G\left(x^{*}, y\right)<0$ for certain $x^{*} \in B_{r}$ and for a positive measure set of $y$. By continuity of $G$ in $x$ we have $G(x, y)<0$ for a (maybe smaller) positive measure set of $y$ and an open set of $x$. Therefore, we can choose a bounded nonnegative function $f$ such that $u(x)=\int_{B_{r}} G(x, y) f(y)<0$ on an open set. But this would contradict to the weak maximum principle, see Corollary 2.1. Thus, we can change $G$ on a null measure set and assume it nonnegative.

Next, suppose that for certain $y^{*}$ the set $\mathcal{S}\left(y^{*}\right)=\left\{x \in B_{r}: G\left(x, y^{*}\right)=0\right\}$ is nonempty. We choose then $x^{0} \in \mathcal{S}\left(y^{*}\right)$ and $y^{0} \in B_{r} \backslash \mathcal{S}\left(y^{*}\right)$ such that $\rho:=\left|x^{0}-y^{0}\right|=$ $\operatorname{dist}\left(y^{0}, \mathcal{S}\left(y^{*}\right)\right)$. Due to the second estimate in Eq. $13 x^{0}$ is separated from $y^{*}$, while $\rho$ can be chosen arbitrarily small. So, we can suppose that

$$
B_{2 \rho}\left(y^{0}\right) \subset B_{r} \backslash\left\{y^{*}\right\} ; \quad B_{\rho / 2}\left(y^{0}\right) \cap \mathcal{S}\left(y^{*}\right)=\emptyset .
$$

We introduce the Green function $\widehat{G}$ of $\mathcal{L}$ in $B_{2 \rho}\left(y^{0}\right)$ and claim that the function $\delta \widehat{G}\left(\cdot, y^{0}\right)$ with sufficiently small $\delta>0$ is a lower barrier for $G\left(\cdot, y^{*}\right)$ in the annulus $\mathcal{X}_{2 \rho, 1 / 4}\left(y^{0}\right)$. Indeed, the boundary $\partial \mathcal{X}_{2 \rho, 1 / 4}\left(y^{0}\right)$ consists of two spheres. Notice that $G\left(\cdot, y^{*}\right)>0$ on $\partial B_{\rho / 2}\left(y^{0}\right)$ while $\widehat{G}\left(\cdot, y^{0}\right)=0$ on $\partial B_{2 \rho}\left(y^{0}\right)$. Thus, there exists a positive $\delta$ such that

$$
G\left(\cdot, y^{*}\right) \geq \delta \widehat{G}\left(\cdot, y^{0}\right) \quad \text { on } \quad \partial \mathcal{X}_{2 \rho, 1 / 4},
$$

and the claim follows. By Lemma $2.4 G\left(\cdot, y^{*}\right) \geq \delta \widehat{G}\left(\cdot, y^{0}\right)$ in the whole annulus, and, in particular,

$$
G\left(x^{0}, y^{*}\right) \geq \delta \widehat{G}\left(x^{0}, y^{0}\right)>0
$$

(the last inequality follows from the second estimate in Eq. 13). The obtained contradiction proves the Lemma.

Proof of Theorem 1.2. We repeat in essential the proof of Lemma 2.5. Denote the set $\mathcal{S}=\{x \in \Omega: u(x)=0\}$ and suppose that $\mathcal{S} \neq \Omega$. Then we can choose $x^{0} \in \mathcal{S}$ and $y^{0} \in \Omega \backslash \mathcal{S}$ such that $\rho:=\left|x^{0}-y^{0}\right|=\operatorname{dist}\left(y^{0}, \mathcal{S}\right)$, and $\rho$ can be chosen arbitrarily small. Repeating the proof of Lemma 2.5 we introduce the same Dirichlet Green function $\widehat{G}\left(\cdot, y^{0}\right)$ of $\mathcal{L}$ in $B_{2 \rho}\left(y^{0}\right)$ and show that $\delta \widehat{G}\left(\cdot, y^{0}\right)$ with sufficiently small $\delta>0$ is a lower barrier for $u$ in the annulus $\mathcal{X}_{2 \rho, 1 / 4}\left(y^{0}\right)$. This ends the proof.

\subsection{The Case $n=2$}

The case $n=2$ is treated basically in the same way as the case $n \geq 3$, but some changes must be done mostly due to the fact that the estimate of the Green function contains logarithm.

Let us explain what changes must be done in the argument in compare with $n \geq 3$. Denote by $G_{0}(x, y)$ the Green function of the problem (9) in the disc $B_{r}$. Then for $x \neq y \in$ $B_{r}$,

$$
\begin{aligned}
& 0<G_{0}(x, y) \leq C_{10}^{\prime} \log \left(\frac{r}{|x-y|}+2\right) ; \\
& \text { if }|x-y| \leq \operatorname{dist}\left(x, \partial B_{r}\right) / 2 \quad \text { then } G_{0}(x, y) \geq C_{20}^{\prime} \log \left(\frac{r}{|x-y|}+2\right),
\end{aligned}
$$

where the constants $C_{10}^{\prime}$ and $C_{20}^{\prime}$ depend only on the ellipticity constants of the operator $\mathcal{L}_{0}$. Indeed by [9] these estimates can be reduced to similar estimates for the Laplacian, when they can be verified directly (in this case the Green function can be written explicitly). 
Analog of Lemma 2.1 reads as follows.

Lemma 2.6 Let $n=2$. There exists a positive constant $R$ depending on the ellipticity constant $v, V M O$-moduli of coefficients $a_{i j}$, the exponent $\alpha$ and the function $\sigma$ from Eq. 7 such that for any $r \leq R$ there is the Green function $G(x, y)$ of the problem (12) in a disc $B_{r} \subset \Omega$. Moreover, it is continuous w.r.t. $x$ for $x \neq y$ and satisfies the estimates

$$
\begin{aligned}
& |G(x, y)| \leq C_{1}^{\prime} \log \left(\frac{r}{|x-y|}+2\right) ; \\
& \text { if }|x-y| \leq \operatorname{dist}\left(x, \partial B_{r}\right) / 2 \quad \text { then }|G(x, y)| \geq C_{2}^{\prime} \log \left(\frac{r}{|x-y|}+2\right),
\end{aligned}
$$

where the constants $C_{1}^{\prime}$ and $C_{2}^{\prime}$ depend on the same quantities as $R$.

To prove this statement we establish the inequality (15) by using the estimate (19) and the assumption (7) instead of Eq. 6. The rest of the proof runs without changes.

Corresponding analog of Lemma 2.2 is true here also.

The remaining part of the proof of Theorem 1.3 is the same as that of Theorem 1.2.

\section{Comparison Theorem for Nonlinear Operators}

\subsection{Proof of Theorem 1.1}

We recall that the statement of Theorem 1.1 with $p>n$ was proved in [7, Theorem 1] by reducing it to the strong maximum principle for the Eq. 3 with continuous leading coefficients and $f\left(x_{1}\right)$ playing the role of a coefficient $b_{1}$. Since the function $f$ depends only on one variable, the assumption $f \in L_{\text {loc }}^{p}(\mathbb{R})$ with a certain $p>1$ implies Eqs. 6 and 7 with $\alpha=p$. Thus, we can apply our Theorems 1.2 and 1.3 (for $n \geq 3$ and $n=2$, respectively) instead of [7, Lemma 1], and the proof of [7, Theorem 1] runs without other changes.

\subsection{Application to Water Wave Theory}

In the paper [7], two theorems were proved on estimates of the free surface profile of water waves on two-dimensional flows with vorticity in a channel, see Theorems 2 and 3 in [7]. The vorticity function $\omega$ in that theorems was assumed to belong to $L_{\text {loc }}^{p}(\mathbb{R})$ with $p>2$ and the proof was based on the application of [7, Theorem 1]. Now the application of our Theorem 1.1 allows us to weaken the apriori assumption for the vorticity function to $\omega \in$ $L_{\text {loc }}^{p}(\mathbb{R}), p>1$.

Acknowledgements V. K. acknowledges the support of the Swedish Research Council (VR) Grant EO418401. A. N. was partially supported by Russian Foundation for Basic Research, Grant 18-01-00472. He also thanks the Linköping University for the hospitality during his visit in January 2018.

Funding Information Open access funding provided by Linköping University.

Open Access This article is licensed under a Creative Commons Attribution 4.0 International License, which permits use, sharing, adaptation, distribution and reproduction in any medium or format, as long as you give appropriate credit to the original author(s) and the source, provide a link to the Creative Commons licence, and indicate if changes were made. The images or other third party material in this article are included in the article's Creative Commons licence, unless indicated otherwise in a credit line to the material. If 
material is not included in the article's Creative Commons licence and your intended use is not permitted by statutory regulation or exceeds the permitted use, you will need to obtain permission directly from the copyright holder. To view a copy of this licence, visit http://creativecommons.org/licenses/by/4.0/.

\section{References}

1. Apushkinskaya, D.E., Nazarov, A.I.: A counterexample to the hopf-oleinik lemma (elliptic case). Anal. PDE 9, 439-458 (2016)

2. Byun, S.-S., Wang, L.: Elliptic equations with BMO coefficients in reifenberg domains. Comm. Pure Appl. Math. 57(N10), 1283-1310 (2004)

3. Davies, E.B., Hinz, A.M.: Kato class potentials for higher order elliptic operators. J. London Math. Soc. 58(2), 669-678 (1998)

4. Gidas, B., Ni, W.-M., Nirenberg, L.: Symmetry and related properties via the maximum principle. Commun. Math. Phys. 68, 209-243 (1979)

5. Grüter, M., Widman, K.-O.: The green function for uniformly elliptic equations. Manuscripta Math 37(N3), 303-342 (1982)

6. Keller, J.B.: On solutions of $\Delta u=f(u)$. Comm. Pure Appl. Math. 10, 503-510 (1957)

7. Kozlov, V., Kuznetsov, N.: A comparison theorem for super- and subsolutions of $\nabla^{2} u+f(u)=0$ and its application to water waves with vorticity. Algebra \& Analysis 30(N3), 112-128 (2018)

8. Kurata, K.: Continuity and harnack's inequality for solutions of elliptic partial differential equations of second order. Indiana Univ. Math. J. 43(N2), 411-440 (1994)

9. Littman, W., Stampacchia, G., Weinberger, H.F.: Regular points for elliptic equations with discontinuous coefficients. Ann. Scuola Norm. Sup. Pisa 17(3), 43-77 (1963)

10. Nazarov, A.I., Uraltseva, N.N.: The harnack inequality and related properties for solutions to elliptic and parabolic equations with divergence-free lower-order coefficients. Algebra \& Analysis 23(N1), 136-168 (2011). (Russian). English transl.: St. Petersburg Math. J. 23 (2012), N1, pp. 93-115

11. Osserman, R.: On the inequality $\Delta u \geq f(u)$. Pacific J. Math. 7, 1641-1647 (1957)

12. Pucci, P., Serrin, J.: The strong maximum principle revisited. J. Diff. Equations 196(N1), 1-66 (2004)

13. Schechter, M.: Spectra of Partial Differential Operators, 2nd edn. North-Holland Series in Applied Mathematics and Mechanics, vol. 14. North-Holland Publishing Co., Amsterdam (1986)

14. Trudinger, N.S.: On Harnack type inequalities and their application to quasilinear elliptic equations. Comm. Pure Appl. Math. 20, 721-747 (1967)

15. Zhang, Q.: A Harnack inequality for the equation $\nabla(a \nabla u)+b \nabla u=0$, when $|b| \in k_{n+1}$. Manuscripta Math 89(N1), 61-77 (1996)

Publisher's Note Springer Nature remains neutral with regard to jurisdictional claims in published maps and institutional affiliations. 